\title{
Acculturation, ethnic identity, and psychological well-being of Albanian-American immigrants in the United States
}

Albina Balidemaj, Rochester Institute of Technology

Mark Small, Clemson University

\section{ABSTRACT}

This study examined the relationship between acculturation, ethnic identity, and psychological wellbeing of the Albanian-American immigrant community in United States. A total of 139 AlbanianAmerican immigrants aged 21-35 years old participated in the study. In order to utilize the data, participants filled out four different surveys, including a demographic questionnaire, the Multigroup Ethnic Identity Measure (MEIM), the Vancouver Index of Acculturation (VIA), and Ryff's

Psychological Well-Being scale. A correlational design relying on cross-sectional survey data and multiple regression analysis was used to study the correlations between acculturation, ethnic identity, and psychological well-being. The results showed that ethnic identity, acculturation, and psychological well-being were positively correlated to each other. In addition, the results showed that both ethnic identity and acculturation affected the psychological well-being of Albanian-

American immigrants in the United States. This relationship was further moderated by gender and length of residency in the United States and mediated through graduate school education. The results of this study will help clinicians, social workers, and policy makers that work with immigrants to better understand the psychological consequences of immigration due to acculturation and ethnic identity factors.

\section{Background}

One of the most recent groups of Europeans to immigrate to the United States are Albanians, although the recorded immigration figures remain small and inaccurate. However, the most recent group of Albanians immigrating to the U.S. was during the 1999 ethnic cleansing of Kosovar Albanians, at which time the United States approved a legal foreign status to about 20,000 Kosovar refugees. According to curren U.S. immigration statistics, overall there are about 250,000 Albanian-Americans residing in the United States (2012). In terms of residency, the 2010 U.S. census shows that the largest number of Albanians live in New York City with a high concentration in the Bronx, followed by Massachusetts, Michigan and New Jersey. According to the National Albanian American Council, family and social life of Albanian-Americans is a fusion of two value systems. The first system belongs to the old, tightly bound Albanians who maintain close contacts with their relatives and continue to preserve the traditional values.

\section{Design and hypotheses}

The first three research questions studied the psychological well-being of the AlbanianAmerican immigrant community in the United examined the correlation between the following factors: acculturation, ethnic identity and psychological well-being of the AlbanianAmerican population in the United States. These factors were chosen for analysis as they have shown significant importance in literature in terms of the overall immigrant psychological well-being. In addition, the fourth research question examined the extent to which acculturation and ethnic identity affect the psychological well-being of Albanian-American immigrants. Lastly, the final research question examined the relationship between the moderating variables of gender, length of residency in the U.S., and ethnicity of the spouse and mediating variables of employment and education. Furthermore, demographic factors such as age, gender, occupation, and length of stay in the country, moderate the relationship between acculturation and psychological wellbeing. States. More specifically, the research questions
Materials

The quantitative survey design consisted of the following standardized scales: A Demographic Questionnaire, The Vancouver Index of Acculturation (VIA), Multigroup Ethnic Identity Measure (MEIM), and The Psychological Wellbeing Inventory (The Ryff Scale)

\section{Procedure}

The participants interested in participating in the study were directed to a web page (hosted via SurveyMonkey) where they completed several questionnaires over the span of approximately 20-30 min.

\section{Results}

The final sample comprised of 139 self-identified Albanian-American adults between the ages 19 and 38, born outside of the United States and associated territories. Specific characteristics of the sample can be seen below:

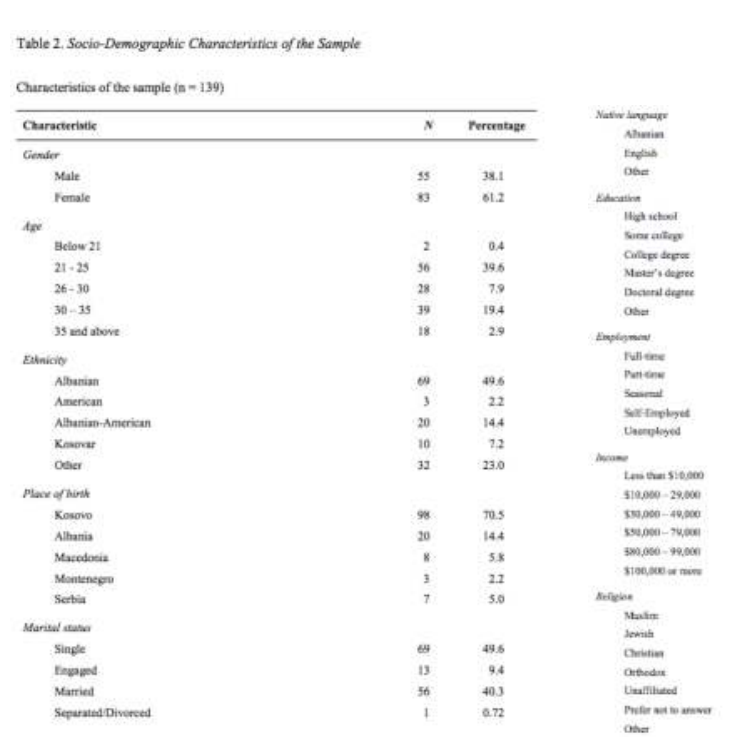

Correlations between acculturation, ethnic identity, and psychological well-being

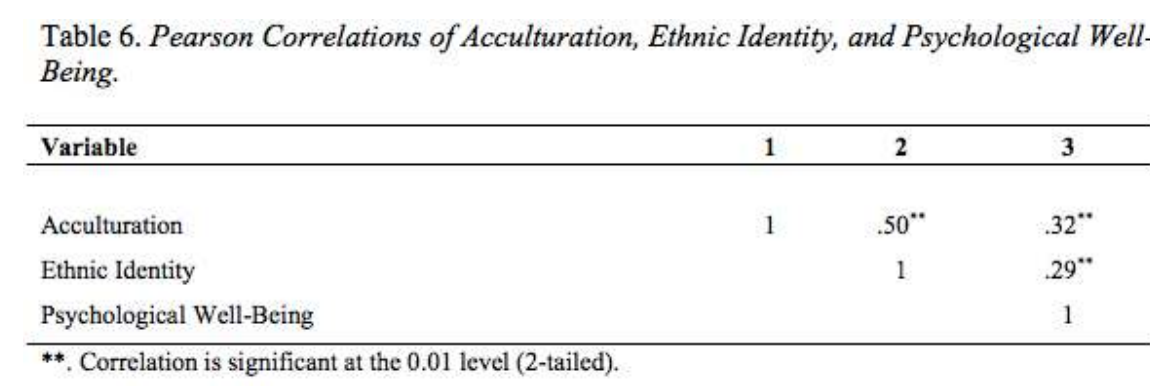

Acculturation and ethnic identity as predictors of psychological well-being

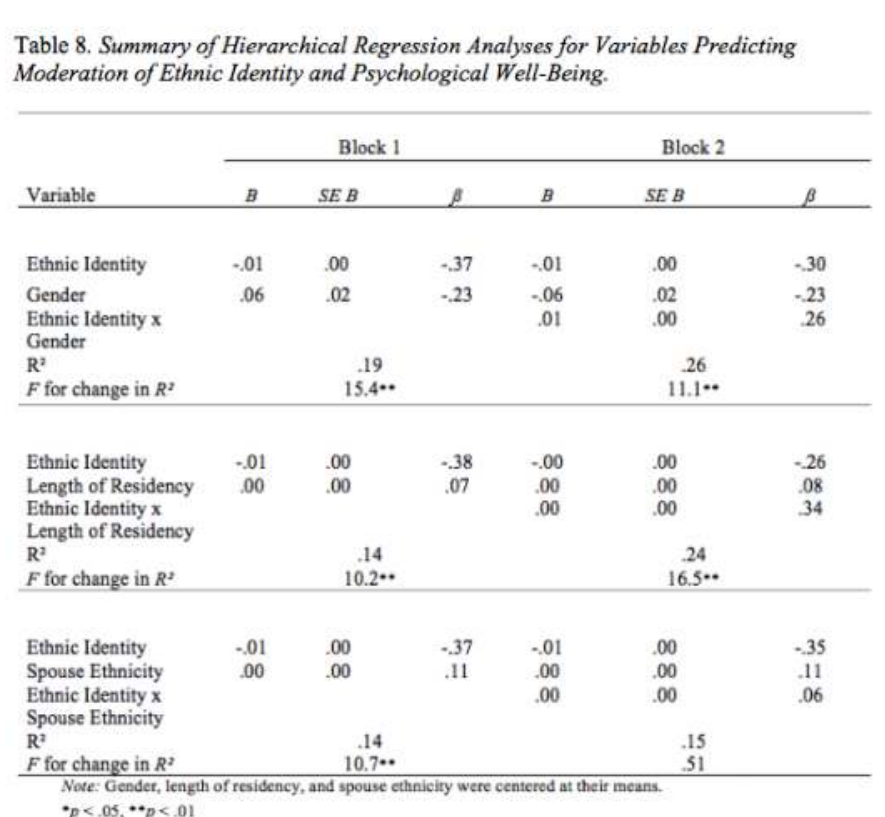

\section{Discussion}

This study examined the correlation between acculturation, ethnic identity and psychological well-being. The results showed that as participants reported higher levels of ethnic identity, they also tended to report higher acculturation levels. In addition, participants reporting higher levels of acculturation reported higher levels of psychological well-being. Finally, as participants reported higher levels of ethnic identity, they also tended to report higher levels of psychological well-being. Higher acculturation and ethnic identity scores were associated with higher scores of psychological well-being for the entire sample.

This study adds to the limited existing literature on immigrant acculturation, ethnic identity, and psychological well-being. Previous research has indicated that the influence of acculturation on psychological well-being shows to be weak or unclear. The results of this study show a rather positive outcome for immigrants. Specialists, researchers, and policy makers may want to focus on the importance of the association between acculturation, ethnic identity, and psychological well-being when designing programs, studies, and policies that aspire to understand and advance the well-being of immigrants. 